\title{
Exploiting V2G to optimize residential energy consumption with electrical vehicle (dis)charging
}

\author{
Kevin Mets, Tom Verschueren, Filip De Turck, Chris Develder \\ Dept. of Information Technology - IBCN, \\ Ghent University - IBBT \\ G. Crommenlaan 8 Bus 201, 9050 Ghent, Belgium \\ Email: \{Kevin.Mets, Chris.Develder\}@intec.ugent.be
}

\begin{abstract}
The potential breakthrough of pluggable (hybrid) electrical vehicles (PHEVs) will impose various challenges to the power grid, and esp. implies a significant increase of its load. Adequately dealing with such PHEVs is one of the challenges and opportunities for smart grids. In particular, intelligent control strategies for the charging process can significantly alleviate peak load increases that are to be expected from e.g. residential vehicle charging at home. In addition, the car batteries connected to the grid can also be exploited to deliver grid services, and in particular give stored energy back to the grid to help coping with peak demands stemming from e.g. household appliances. In this paper, we will address such so-called vehicle-to-grid (V2G) scenarios while considering the optimization of PHEV charging in a residential scenario.

In particular, we will assess the optimal car battery (dis)charging scheduling to achieve peak shaving and reduction of the variability (over time) of the load of households connected to a local distribution grid. We compare (i) a business-as-usual (BAU) scenario, without any intelligent charging, (ii) intelligent local charging optimization without V2G, and (iii) charging optimization with V2G. To evaluate these scenarios, we make use of our simulation tool, based on OMNeT++, which combines ICT and power network models and incorporates a Matlab model that allows e.g. assessing voltage violations. In a case study on a threefeeder distribution network spanning 63 households, we observe that non-V2G optimized charging can reduce the peak demand compared to BAU with $64 \%$. If we apply V2G to the intelligent charging, we can further cut the non-V2G peak demand with $17 \%$ (i.e., achieve a peak load which is only $30 \%$ of BAU).
\end{abstract}

\section{INTRODUCTION}

Plug-in-(hybrid)-electric vehicles (PHEVs) will represent a significant new load on the existing distribution grids, especially as their penetration level increases (cf. the much debated goal in US of having one million plug-in EVs by 2015 [1]). Indeed, charging a single EV at home, for the average household means a doubling of the average load [2]. These changes in load patterns may require upgrades to (distribution) power grid components such as transformers. Uncoordinated charging also has an impact on the performance of the distribution grid in terms of power losses and power quality [3]. Hence, the charging of these vehicles must carefully be managed to avoid overloads or other power grid problems, by for example shifting the charging in time. This is possible, as personal vehicles are only used $4 \%$ of the time for transportation, and the remaining $96 \%$ can be used for other purposes [4].

However, PHEVs can also be of benefit towards the power grid, for example to store renewable energy which is inter- mittent by nature. If power generation becomes increasingly dependent on such renewable sources, supply and demand matching will obviously become more challenging [5]. Exploiting the flexibility in deciding when to charge a PHEV battery can partly alleviate this problem of intermittent (and unpredictable) energy supply. Moreover, the batteries may also be exploited as temporary storage of the fluctuating energy supply, and serve as energy storage resource that can give energy back to the grid while parked, also known as vehicleto-grid (V2G) power [4]. Thus, not only can the renewable energy be used to power the transport functions of the PHEV, but V2G can also be exploited to deliver applications to the grid (peak power, spinning reserves, regulation, etc.).

Note that intelligent control strategies for (dis)charging EVs is part of a broader context called demand-side management (DSM), which is an active research topic. e.g., [6] proposes a distributed DSM approach based on game-theoretic energy scheduling (to reduce energy costs and the peak-to-average ratio, while maintaining privacy). Multi-agent systems based on virtual markets such as PowerMatcher [7] are another approach to DSM, where agents bid on an electronic market to determine an equilibrium price matching demand and supply.

In this paper, we will assess the optimal peak load reduction as well as the flatness of the load profile that can be achieved by intelligently scheduling EV battery charging. We investigate the maximal reduction achievable by an allknowing scheduling algorithm that has full knowledge of the energy consumption (load profile) of a household, the arrival and departure times of an EV and its state-of-charge. We will compare scheduling with/without V2G and a baseline scenario without any intelligent charging. Thus, we set the performance boundaries of the peak load reduction and load flattening which would be achievable by any real-time, local control mechanism (such as aforementioned DSM approaches). To determine these boundaries in a case study, we use quadratic programming and our simulation tool [8].

The remainder of this paper is structured as follows: our problem statement is summarized in Section II. A brief overview of our simulation tool is listed in Section III. The mathematical formulation of the resulting optimization problem is detailed in Section IV. In Section V, we present the results of our case study comprising 63 households. Finally, conclusions and future work are synthesized in Section VI. 


\section{APPROACH AND PROBLEM STATEMENT}

Our earlier work [9] indicated that a distribution transformer's peak load caused by uncontrolled charging can be greatly reduced through local or iterative global energy control of the charging process of EVs at households. In particular, we proposed a local and a global control strategy to coordinate the PHEV charging process, comparing them to a business-asusual (BAU) scenario where charging starts uncontrolled, as soon as the vehicles are plugged in upon arrival at home. The local control algorithm only considers the current household, to decide upon when to charge the car arriving there. Thus, we assess the possible advantage that could be offered by a so-called home energy controller, that takes decisions based on knowledge of the household energy consumption (e.g. exploiting historical data gathered by the local meter, and associated forecasts). The global control approach relied on sharing knowledge between the various homes (thus requiring communication and coordination between them), to try and make globally optimal decisions.

When compared to BAU, the global method results in the largest reduction of both the peak load $(30 \%$ reduction in a scenario with $30 \%$ PHEVs) and load profile variability (75\% reduction of the standard deviation between the values of the load profile in the same scenario). The local algorithm only performed slightly less good: $26 \%$ peak reduction and $58 \%$ reduction in load profile variability for $30 \%$ PHEV penetration. Even though the local algorithm is suboptimal (compared to the global method), it achieves a significant improvement compared to BAU. Note that such a local algorithm also would not necessarily require communication, or at least no exchange of data pertaining to other households. The latter is an important privacy concern.

Based on these considerations, we have opted in the current paper to further study the local control approach. We here present an extension of our local algorithm to cater for V2G: energy stored in the battery can later be fed back for local energy consumption within the household, thus further reducing the household peak load and load profile variability. We thus will compare local strategies with/without V2G.

The general problem we consider is the following:

\section{Given}

- Grid topology comprising the houses and the distribution grid interconnecting them;

- Household loads, specifying the electrical loads within the home, i.e. appliances, HVAC, etc.; and

- PHEV status, including the arrival and departure times, as well as state-of-charge;

\section{Find}

- when to charge the PHEV battery;

- if and when to decharge that battery for V2G operation;

Such that the peak net energy consumption, as well as its variability over time, is minimized.

We will model residential energy consumption as consisting of two parts: uncontrollable and controllable loads. Uncontrollable loads cannot be shifted in time and their energy consumption cannot be changed. Each household is characterized by a load profile for the uncontrollable loads that indicates the average uncontrollable load at each time of day. Controllable loads on the other hand can be shifted in time and their energy consumption can be changed. In this work, we only consider EVs as controllable loads. Note that, when considering V2G applications, the electric vehicles are not only considered as loads, but also as producers. Hence, we assume EVs to be controllable loads as well as controllable producers: at any given moment, a car's battery will either be charged, discharged, or left untouched.

We will determine a combined charging and discharging schedule. We consider the scheduling to operate for discrete time intervals, i.e. for time slots of equal size. The algorithm determines during which time slots an EV should charge or discharge and the rate at which this happens. Since we are focusing on a local approach, only knowledge of local power consumption is used. Also, the impact of other households and vehicles on the global load profile is not considered when determining the (dis)charging schedules. Therefore, the objective of the schedule will be to minimize local peak load and load profile variability. Clearly, the intention - which [9] proved to be achievable - is to affect the aggregate load profile comprising all households connected to a single distribution transformer. Even though the quadratic programming model described below is solved for each vehicle separately upon arrival at the charging point, we will assess its impact by showing the total load on the whole distribution feeders.

Note that we assumed a simplified, lossless model of the car's battery, which we assume to be characterized by (i) maximum storage capacity, and (ii) maximum charge/decharge power. For simplicity, in this work we disregard any battery inefficiencies such as self-discharge, loss of capacity (e.g. due to memory effects), etc.

\section{Simulation TOOL}

The smart grid simulator we have developed to facilitate smart grid research, combines models of the power grid itself as well as the information and communication technologies (ICT) that will be deployed in the smart grid. The main goals of our smart grid simulation environment are:

- Support the development of control algorithms for energy management and their corresponding software architectures.

- Enable analysis of communication requirements and impact of specific communication technologies on the performance of control algorithms.

- Enable evaluation of the impact of the control strategies on the power grid.

- Provide a flexible and modular environment.

To achieve these goals, we based our tool on $\mathrm{OMNeT}++$, given its excellent performance [10]. For the assessment of power network characteristics, we integrated a Matlab module, based on the fast harmonic simulation method presented in [11]. For an overview of our simulator's architecture, we refer to [8]. 


\section{OPTIMIZATION MODEL}

The simulated time (e.g. 24 hours) is divided into equal time slots of duration $\Delta t$ (e.g. $\Delta t=5$ minutes). The algorithm determines during which time slots the vehicle can charge or discharge and also the rate at which this happens. We assume there are $K$ households at which at most one PHEV is present and that the variable $k \in\{1, \ldots, K\}$ indicates a specific household. The arrival time of the vehicle of household $k$ is indicated by $\alpha_{k}$ and the departure time by $\beta_{k}$. The maximum battery capacity (expressed in $\mathrm{kWh}$ ) is specified by $C_{k, \max }$ and the energy stored (kWh) in the battery upon arrival is specified by $C_{k}$. The following optimization model is solved for each household with an electric vehicle.

A target load profile is calculated before determining the charging schedule. The goal is for the household load profile, which includes the uncontrollable load and charger load, to approach this target profile as closely as possible. First, we determine which constant average power $B L_{k}(\mathrm{~kW})$ would be required to provision the uncontrollable loads when the vehicle is at home. $B_{k}(t)$ indicates the uncontrollable load $(\mathrm{kW})$ consumed during time slot $t$.

$$
B L_{k}=\frac{\sum_{t^{\prime}=\alpha_{k}}^{\beta_{k}} B_{k}\left(t^{\prime}\right)}{\beta_{k}-\alpha_{k}}
$$

Next, we determine which constant load $C L_{k}$ would be required to charge the battery, assuming the charging process is started upon arrival and stopped on departure. A conversion factor $\delta=\frac{60}{\Delta t}$ is used to convert between energy (kWh) used during a time slot and instantaneous electrical power $(\mathrm{kW})$ required therefore.

$$
C L_{k}=\frac{\left(C_{k, \max }-C_{k}\right) \cdot \delta}{\beta_{k}-\alpha_{k}}
$$

The optimal load profile, considering the goals of minimizing the peak load and load profile variability, is formed by a constant load which is defined as the sum of the constant uncontrollable loads and the controllable loads. $T_{k}(t)$ is the constant power $(\mathrm{kW})$ that should be supplied during $\left[\alpha_{k}, \beta_{k}\right]$ to satisfy the total net power demand in that period, i.e. the demand we could achieve if we are able to perfectly spread the energy demand.

$$
T_{k}(t)=B L_{k}+C L_{k}
$$

The decision variables $X_{k}(t)$ of the optimization problem form the charging schedule and indicate the charing or discharging rate during each time slot. The vehicle is charging when $X_{k}(t)>0$ and is discharging when $X_{k}(t)<0$. The charger is disabled when $X_{k}(t)=0$

$$
\begin{aligned}
X_{k}(t) \in\left[-X_{k, \max }, X_{k, \max }\right] \quad, \quad \forall t \in\left\{\alpha_{k}, \ldots, \beta_{k}\right\} \\
X_{k}(t)=0 \quad, \quad \forall t \notin\left\{\alpha_{k}, \ldots, \beta_{k}\right\}
\end{aligned}
$$

Equation 6 assures that the load of the household does not exceed the the maximum load $L_{k, \max }$ (expressed in $\mathrm{kW}$ ) of the household connection.

$$
B_{k}(t)+X_{k}(t) \leq L_{k, \max }
$$

$C_{k, \max }$ indicates the maximum battery capacity (expressed in $\mathrm{kWh}$ ), and equation 7 assures that the battery is fully charged after applying the charging schedule.

$$
C_{k}+\sum_{t=\alpha_{k}}^{\beta_{k}}\left(X_{k}(t) \cdot \delta\right)=C_{k, \max }
$$

These constraints (however with the interval in equation 4 limited to $\left[0, X_{k, \max }\right]$ ) would suffice when only considering charging. However, as we also consider discharging, we have to add more constraints to the optimization model to make sure that the battery is not overcharged or discharged too low. Therefore, we define the current battery capacity at the end of time slot $t\left(\alpha_{k} \leq t \leq \beta_{k}\right)$ as:

$$
C_{k}(t)=C_{k}+\sum_{t^{\prime}=\alpha_{k}}^{t}\left(X_{k}\left(t^{\prime}\right) \cdot \delta\right)
$$

To avoid overcharging the battery, we add the following constraint, which states that at no moment in time, the stored energy in the battery can not exceed the maximum battery capacity.

$$
\forall i \in\left\{\alpha_{k}, \ldots, \beta_{k}\right\}: C_{k}(i) \leq B C_{k}
$$

To avoid discharging the battery too low we add the following constraint, which states that at no moment in the time, the energy stored in the battery can go below a certain threshold, which is assumed to be zero.

$$
\forall i \in\left\{\alpha_{k}, \ldots, \beta_{k}\right\}: C_{k}(i) \geq 0
$$

The objective function is defined in equation 11. A charging schedule is obtained by minimizing the Squared Euclidean Distance between the target load profile and the household load profile. The goal is to reduce the peak load and load profile variability of the household load profile by controlling the charging process. By shifting the charging process in time and controlling the charging speed, we can reduce the impact on the load profile. However, by also considering discharging, we can perform peak shaving where the electricity for the peak load of the household is provided by the battery of the vehicle, therefore reducing the load on the power grid.

$$
\sum_{t=\alpha_{k}}^{\beta_{k}}\left(T_{k}(t)-\left(B_{k}(t)+X_{k}(t)\right)^{2}\right.
$$

\section{CAse Study}

\section{A. Simulation parameters}

We assume a residential area of 63 households. Each household is randomly assigned a load profile from a set of five load profiles obtained from measurements of Belgian households during different winter days. To avoid artificial synchronization, each household load profile is shifted in time using a uniform distribution between 0 and 60 minutes. We assume a standard connection to the grid of $230 \mathrm{~V}$ and $40 \mathrm{~A}$ resulting in a line limit of $9.2 \mathrm{~kW}$; household power demand must remain below this limit (which is the case in Belgium for standard residential connections). We consider a three phase 
distribution grid consisting of three feeders of equal length. 21 households are connected to each feeder with equal distances between them, and the household connections (single phase) are uniformly distributed over the three phases.

We evaluate three penetration degrees of PHEV, in which $15 \%, 45 \%$ and $75 \%$ of the households own a PHEV, and assume that at any time there is at most one PHEV at each household. Two types of battery are considered, a $15 \mathrm{kWh}$ battery simulating a PHEV, and a $25 \mathrm{kWh}$ battery simulating an EV. Also, two types of charger are considered, a slow charger of $3.6 \mathrm{~kW}$ and a fast charger of $7.4 \mathrm{~kW}$. Combining these options, we obtain four types of vehicles that are simulated in the experiments. The amount of each type of vehicle in each scenario is shown in Table I. A random state of charge between $20 \%$ and $60 \%$ is assigned to each vehicle using a uniform distribution. The plug-in and plug-out times are randomly selected around 17:30 and 06:30 using a normal distribution with a standard deviation of 45 minutes.

\section{B. Results and discussion}

We have performed hundred simulations for each scenario using different seeds for the random number generators. We compared the results of the smart charging algorithms with the results of a reference BAU scenario in which vehicles are charged immediately when plugged in using a constant charging rate of $3.6 \mathrm{~kW}$ or $7.4 \mathrm{~kW}$. Figure 1 illustrates the power demand of a single household and the impact that the local algorithm with vehicle-to-grid has on it. A peak load is observed at 18:00 originating from uncontrollable loads (e.g. cooking equipment). As the vehicle arrives, there is still energy stored in the battery, which is supplied to the household. The uncontrollable loads use this energy instead of drawing it from the grid. As a result, the power demand towards the power grid is reduced, as can be seen by the total load profile. However, if no vehicle-to-grid power would be supplied to the household, it would not be possible to reduce the peak demand towards the grid, as there is no control over the uncontrollable loads. The other peak loads around the interval 08:00-10:00 are not reduced because we have assumed that the vehicle should be available for use during that time period.

Figure 2 illustrates the demand at the level of the distribution transformer, thus the net power supplied to all households together, obtained from performing the different strategies. The graphs shown are averages obtained from hundred independent simulation runs. It is clear that by using vehicle-to-grid power

Table I

AMOUNT OF PHEV AND EV AND THEIR TYPE OF BATTERY CHARGER IN THE THREE DIFFERENT SCENARIOS.

\begin{tabular}{|l||c|c|c|c|}
\hline $\begin{array}{l}\text { Scenario } \\
\text { (\% penetration) }\end{array}$ & $\begin{array}{c}\text { PHEV } \\
3.6 \mathrm{~kW}\end{array}$ & $\begin{array}{c}\text { PHEV } \\
7.4 \mathrm{~kW}\end{array}$ & $\begin{array}{c}\text { EV } \\
3.6 \mathrm{~kW}\end{array}$ & $\begin{array}{c}\mathrm{EV} \\
7.4 \mathrm{~kW}\end{array}$ \\
\hline Light (15\%) & 4 & 3 & 2 & 1 \\
\hline Medium (45\%) & 10 & 10 & 5 & 4 \\
\hline Heavy (75\%) & 17 & 16 & 7 & 7 \\
\hline
\end{tabular}

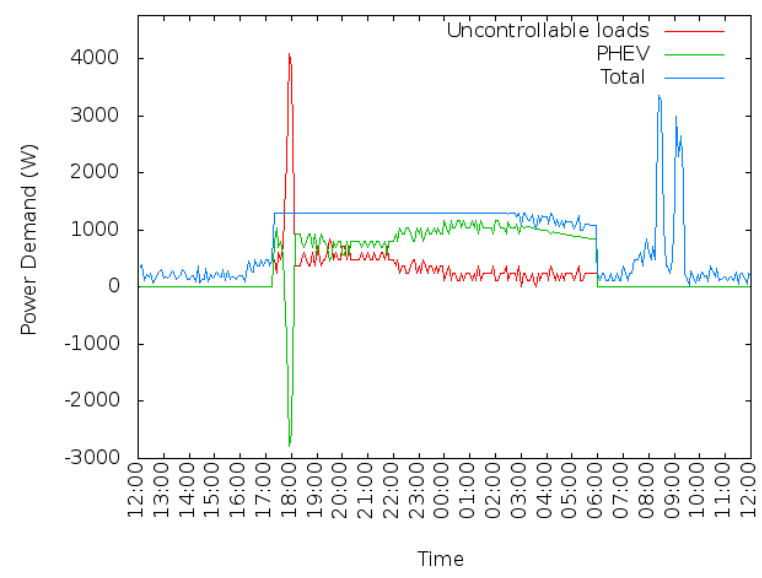

Fig. 1. Example of the impact of the Local+V2G control strategy on a single household.

Table II

IMPACT OF THE CONTROL STRATEGIES ON THE PEAK LOAD OF THE DISTRIBUTION TRANSFORMER LOAD PROFILE. RESULTS ARE AVERAGES OVER 100 SIMULATIONS PERFORMED FOR EACH SCENARIO. THE STANDARD DEVIATION IS GIVEN IN PARENTHESIS.

\begin{tabular}{|l|c|c|c|}
\hline & \multicolumn{3}{|c|}{ PHEV penetration level } \\
\hline & Light (15\%) & Medium (45\%) & Heavy (75\%) \\
\hline \hline BAU & $121 \mathrm{~kW}(7.6)$ & $199 \mathrm{~kW}(12.9)$ & $274 \mathrm{~kW}(17.1)$ \\
\hline Local & $86 \mathrm{~kW}(5.0)$ & $94 \mathrm{~kW}(4.5)$ & $100 \mathrm{~kW}(4.4)$ \\
\hline Local+V2G & $82 \mathrm{~kW}(4.8)$ & $84 \mathrm{~kW}(4.6)$ & $83 \mathrm{~kW}(4.4)$ \\
\hline
\end{tabular}

for local residential energy consumption, we can further reduce the evening peak load. Instead of demanding power from the grid, power is provided by the batteries of the plug-in-(hybrid)electric vehicles to the appliances over which no control can be executed, thereby lowering the demand for grid power. Table II gives more detailed information regarding the reduction of the peak load for each scenario and algorithm. Uncontrolled charging leads to an average peak load of $121 \mathrm{~kW}, 199 \mathrm{~kW}$ and $274 \mathrm{~kW}$ respectively for the $15 \%, 45 \%$, and $75 \%$ scenarios. The local algorithm without $\mathrm{V} 2 \mathrm{G}$ power results in an average peak load of $86 \mathrm{~kW}, 94 \mathrm{~kW}$, and $100 \mathrm{~kW}$, or a reduction of respectively $29 \%, 53 \%$, and $64 \%$. When V2G power is used, we obtain lower average peak loads, respectively $82 \mathrm{~kW}, 84$ $\mathrm{kW}$, and $83 \mathrm{~kW}$, or a reduction of respectively $32 \%, 58 \%$, and $70 \%$ compared to the reference BAU case. When we compare the local algorithm that uses $\mathrm{V} 2 \mathrm{G}$ power to the algorithm without $\mathrm{V} 2 \mathrm{G}$, we obtain a reduction of $4 \%, 10 \%$, and $17 \%$ for the different scenarios. Thus the impact of V2G is nonnegligible, even in this otherwise fairly deterministic scenario.

We are not only concerned about the peak load of the distribution transformer, but also in the variability of the load profile over time: ideally, we'd like to see a fairly flat load profile. We evaluate the variability of the load profile using the standard deviation between the values of the load profile. Table III gives an overview of the results. Uncontrolled charging results in a distribution transformer load profile with a 
Table III

IMPACT OF THE CONTROL STRATEGIES ON THE VARIABILITY OVER TIME (I.E. STANDARD DEVIATION) OF THE DISTRIBUTION TRANSFORMER LOAD PROFILE. THE STANDARD DEVIATIONS FOR THESE VALUES ARE ALSO

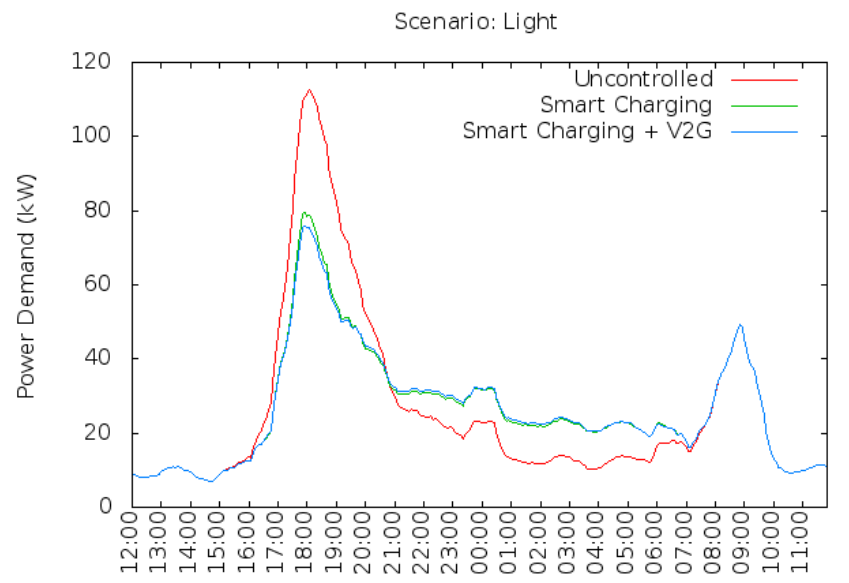

Time
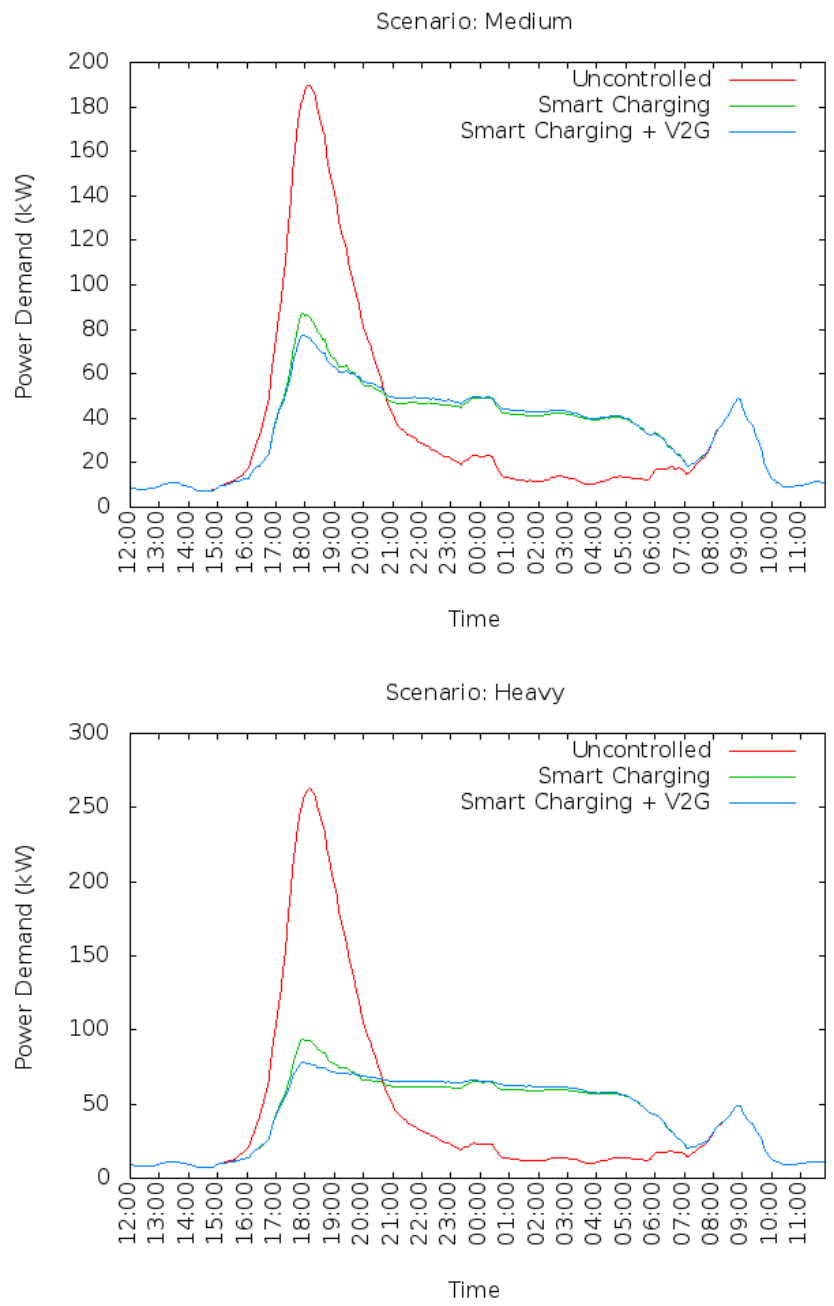

Fig. 2. Average load profiles as would be measured by the distribution transformer, obtained from executing each scenario hundred times using different random seeds. GIVEN IN PARENTHESIS.

\begin{tabular}{|l|c|c|c|}
\hline & \multicolumn{3}{|c|}{ PHEV penetration level } \\
\hline & Light (15\%) & Medium (45\%) & Heavy (75\%) \\
\hline \hline BAU & $24.9 \mathrm{~kW}(0.8)$ & $43.9 \mathrm{~kW}(1.7)$ & $62.3 \mathrm{~kW}(2.0)$ \\
\hline Local & $16.2 \mathrm{~kW}(0.2)$ & $19.8 \mathrm{~kW}(0.4)$ & $24.9 \mathrm{~kW}(0.5)$ \\
\hline Local+V2G & $15.7 \mathrm{~kW}(0.3)$ & $19.0 \mathrm{~kW}(0.4)$ & $24.3 \mathrm{~kW}(0.6)$ \\
\hline
\end{tabular}

standard deviation between the values of $24.90 \mathrm{~kW}, 43.95 \mathrm{~kW}$, and $62.30 \mathrm{~kW}$ for the $15 \%, 45 \%$ and $75 \%$ scenarios. These results indicate that uncontrolled charging and an increasing number of vehicles leads to more variability. By performing local energy control (without V2G power) we can achieve standard deviations of $16.16 \mathrm{~kW}, 19.76 \mathrm{~kW}$, and $24.92 \mathrm{~kW}$, or a reduction of respectively $35 \%, 55 \%$, and $60 \%$ compared to the uncontrolled scenarios. When V2G power is used, the standard deviations are lowered to $15.70 \mathrm{~kW}, 18.99 \mathrm{~kW}$, and $24.29 \mathrm{~kW}$, or a reduction of $37 \%, 57 \%$, and $61 \%$ compared to the uncontrolled BAU scenarios. Not only does using V2G power reduce the peak load, it also reduces the variability of the load profile.

We are also concerned with the impact these different strategies have on the voltages within the distribution grid. According to the EN50160 standard, the voltage should be $230 \mathrm{~V} \pm 10 \%$. Therefore, we have performed a detailed power flow simulation for a scenario with high peak loads. We have chosen the scenario with a PHEV penetration level of $45 \%$. Figure 3 gives an overview of the results obtained. The graphs give for each time slot the number of households where a voltage deviation larger then $10 \%$ is observed. It is clear that uncontrolled charging leads to a large number of voltage deviations. These deviations occur when the highest loads are observed. However, by performing local energy control (without V2G power), this number is drastically lowered. When we perform local energy control with V2G power, we no longer observe any voltage deviations larger then $10 \%$.

\section{CONCLUSION}

In this work we evaluated the impact of using vehicle-togrid (V2G) power in a local control algorithm for managing the charging of electric vehicles. Simulation results for three control strategies were compared: uncontrolled charging (BAU), local control without V2G, and local control with V2G. We evaluated the performance of these strategies in terms of distribution transformer peak load and load profile variability for a residential area comprising 63 households and different penetration levels of PHEVs (15\%, 45\% and 75\% of the households). Simulation results showed that the local algorithm reduces the peak load that stems from uncontrolled charging by respectively $29 \%, 53 \%$, and $64 \%$ for the three penetration levels. However, when V2G power is used, we obtain an additional peal load reduction of $4 \%, 10 \%$, and 


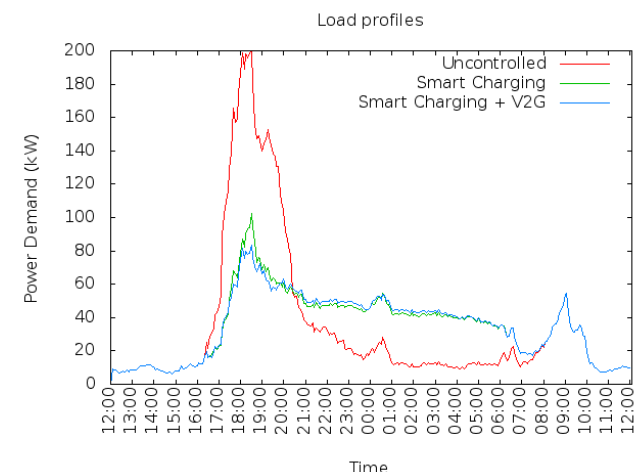

(a) Load profiles for the different strategies. A single scenario with a PHEV penetration degree of $45 \%$ and high peak loads was chosen for the analysis.

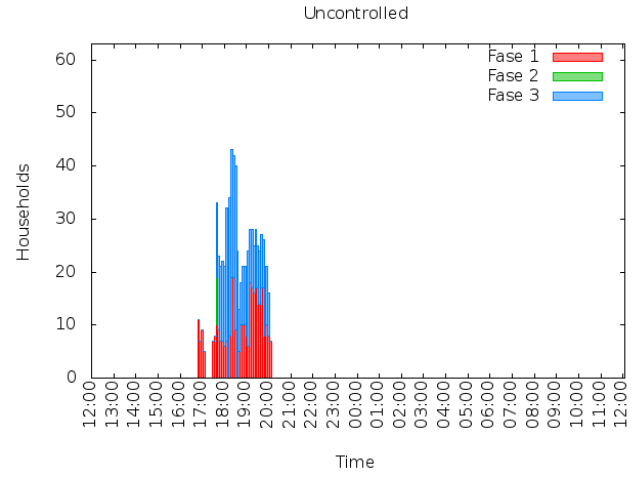

(b) Number of households where voltage deviations larger then $10 \%$ are observed for the uncontrolled (BAU) charging case.

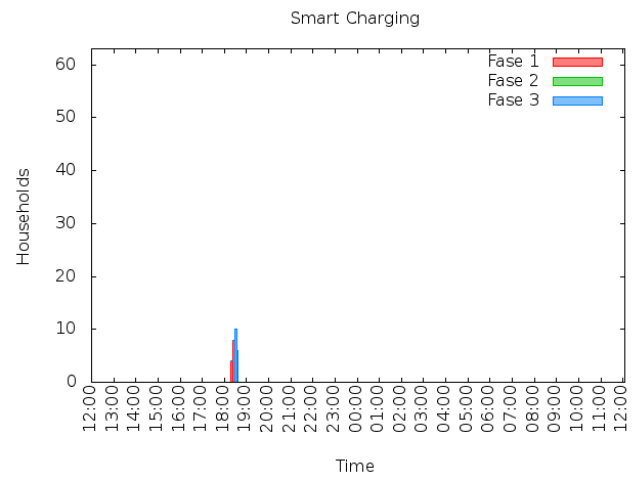

(c) Number of households where voltage deviations larger then $10 \%$ are observed for the smart charging case without $\mathrm{V} 2 \mathrm{G}$ power.

Fig. 3 .

$17 \%$ when compared to local control without V2G power. Local control using V2G power also reduces the variability of the load profile with respectively $37 \%, 57 \%$ and $61 \%$ compared to BAU. Uncontrolled charging also results in a large number of voltage deviations of more than $10 \%$ from the nominal voltage. However, local energy control significantly reduces this number, and when $\mathrm{V} 2 \mathrm{G}$ power is used no voltage deviations are observed. These results show that using V2G power can improve the results of a local energy control strategy.
Ongoing and future work includes, as a first step, to explore the global optimum that can be reached in case of $\mathrm{V} 2 \mathrm{G}$, thus basing the optimization on overall knowledge of all household loads and their cars. Next steps comprise the development of online/distributed implementations of the control strategy (e.g. price-based), and assess how well they approach the boundaries found by our all-knowing optimization. In addition, we will further identify the impact on the distribution work in terms of e.g. voltage violations, power quality etc. especially when considering also renewable energy sources (such as photo-voltaic panels installed in the homes [12]) and the opportunities of exploiting V2G to counter those power issues. Complementary work involves judging, and possibly minimizing, the impact the increased number of charging and discharging cycles has on the lifetime of the batteries.

\section{ACKNOWLEDGMENT}

Work presented in this paper has been supported by the Flemish Government through the LINEAR project (through IWT) and the project SmartE (an IBBT ICON project). K. Mets would like to thank the Agency for Innovation by Science and Technology in Flanders (IWT) for financial support through his Ph.D. grant. C. Develder is partially supported by the Research Foundation - Flanders (FWO Vlaanderen) as a post-doctoral fellow.

\section{REFERENCES}

[1] J. Voelcker, "One million plug-in cars by 2015?" IEEE Spectrum, vol. 48 , no. 4 , pp. 11-13, Apr. 2011

[2] A. Ipakchi and F. Albuyeh, "Grid of the future," IEEE Power and Energy Magazine, vol. 7, no. 2, pp. 52-62, Mar.-Apr. 2009.

[3] K. Clement-Nyns, E. Haesen, and J. Driesen, "The impact of charging plug-in hybrid electric vehicles on a residential distribution grid," IEEE Trans. Power Sys., vol. 25, no. 1, pp. 371-380, Feb. 2010.

[4] W. Kempton and J. Tomic, "Vehicle-to-grid power fundamentals: Calculating capacity and net revenue," J. Power Sources, vol. 144, no. 1, pp. 268-279, Jun. 2005.

[5] - "Vehicle-to-grid power implementation: From stabilizing the grid to supporting large-scale renewable energy," J. Power Sources, vol. 144, no. 1, pp. 280-294, Jun. 2005.

[6] A. Mohsenian-Rad, V. Wong, J. Jatskevich, R. Schober, and A. LeonGarcia, "Autonomous demand-side management based on gametheoretic energy consumption scheduling for the future smart grid," IEEE Trans. Smart Grid, vol. 1, no. 3, pp. 320-331, Dec. 2010.

[7] J. K. Kok, C. J. Warmer, and I. G. Kamphuis, "PowerMatcher: Multiagent control in the electricity infrastructure," in Proc. 4th Int joint Conf. Autonomous Agents and Multiagent Systems, New York, NY, USA, 2005, pp. 75-82.

[8] K. Mets, T. Verschueren, C. Develder, T. Vandoorn, and L. Vandevelde, "Integrated simulation of power and communication networks for smart grid applications," in Proc. 16th IEEE Int. Workshop Computer Aided Modeling, Analysis and Design of Commun. Links and Netw. (CAMAD 2011), Kyoto, Japan, 10-11 Jun. 2011, pp. 61-65.

[9] K. Mets, T. Verschueren, W. Haerick, C. Develder, and F. De Turck, "Optimizing smart energy control strategies for plug-in hybrid electric vehicle charging," in Proc. 1st IFIP/IEEE Int. Workshop on Management of Smart Grids, at 2010 IEEE/IFIP Netw. Operations and Management Symp. (NOMS 2010), Osaka, Japan, 19-23 Apr. 2010, pp. 293-299.

[10] E. Weingärtner, H. Vom Lehn, and K. Wehrle, "A performance comparison of recent network simulators," in Proc. IEEE Int. Conf. Commun. (ICC 2009), Dresden, Germany, 14-18 Jun. 2009, pp. 1287-1291.

[11] L. Degroote, L. Vandevelde, and B. Renders, "Fast harmonic simulation model for the analysis of network losses with converter-connecter distributed generation," Electric Power System Research, vol. 80, no. 11, pp. 1332-1340, Nov. 2010.

[12] T. Verschueren, K. Mets, M. Strobbe, B. Meersman, C. Develder, and L. Vandevelde, "Assessment and mitigation of voltage violations by solar panels in a residential distribution grid," in Proc. 2nd IEEE Int. Conf. Smart Grid Communications (SmartGridComm 2011), Brussels, Belgium, 17-20 Oct. 2011, to appear. 